\title{
On Intuitionistic Fuzzy Metric Neighbourhoods
}

\author{
Evgeniy Marinov ${ }^{1}$ Peter $\operatorname{Vassilev}^{1}$ Krassimir Atanassov $^{1}$ \\ ${ }^{1}$ Department of Bioinformatics and Mathematical Modelling \\ Institute of Biophysics and Biomedical Engineering \\ Bulgarian Academy of Sciences \\ 105 Acad. G. Bonchev Str., Sofia 1113, Bulgaria \\ e-mails: evgeniy.marinov@biomed.bas.bg, peter.vassilev@gmail.com, krat@bas.bg
}

\begin{abstract}
The concept of an intuitionistic fuzzy neighbourhood is introduced. This notion turns out to be more sensitive to variations in the different points of the universe compared to the standard neighbourhood definition of metric space. We apply the intuitionistic fuzzy neighbourhood to the extended modal operators, defined over intuitionistic fuzzy sets.
\end{abstract}

Keywords: Intuitionistic fuzzy set, Neighbourhood, Topology.

\section{Introduction}

The point of departure of this work is the concept of distances between Intuitionistic Fuzzy Sets (IFSs), (see $[3,4])$. They constitute an important extension of the concept of Zadeh's fuzzy sets (cf. [15]) that can model uncertain and imprecise information when the evaluations are concerned with a bipolar type of evidence. The generalization here is that the "pro" and "contra" estimations do not sum to one (truth) but there is a degree of uncertainty. In the context of intuitionistic fuzzy sets many distance measures have been proposed, notably those which are counterparts of the respective distance measures proposed for fuzzy sets $[1,5,13]$. Our point of departure is the standard definition of neighbourhood for metric spaces (taking the base of open balls to construct the metric topological spaces) in the framework of the already proposed metrics for IFSs. We introduce here a new type of neighbourhood, called IF-neighbourhood and IF-ball, respectively. A few interesting theorems and the connection to the standard metric neighbours are also stated. We propose an application of the developed theory and definitions using the extended modal operators for IFSs.

The paper is organized as follows. In Section 2 we introduce the notions of topological spaces and metric spaces (distances) in general, whereas in Section 3 are discussed the distances on IFSs. In Section 4 are provided the main results of the current work and in Section 5 are the applications to the proposed theory.

\section{Topological Metric Spaces and Neighbourhoods}

Topology is one of the most important areas of mathematics and its applications (cf. [2,7]). Many mathematicians and scientists actively employ concepts of topology to model and understand realworld structures and problems. A rich variety of results also has emerged in other areas of applied mathematics stemming from pure topological research. Topology is often described as a rubbersheet geometry - the study of position or location of points (elements) belonging to a given set called topological space. In traditional geometry, objects such as circles, polyhedra, triangles, etc., are considered as rigid figures (bodies), with well-defined distances between points and angles between edges or faces. One of the most useful types of topological spaces emerges when along with the common properties the notion of distance or metric between objects of the underlying space is defined. This type of topology we are going to employ in the current paper.

In what follows with $\mathcal{P}(X)=\{A \mid A \subset X\}$ or $2^{X}=\{\mu: X \rightarrow\{0,1\}\}$ we will denote the set of subsets of any set $X$. In fact the above defined concepts are bijective, as one can easily see by taking the mapping $A=\mu^{-1}(1)$. Let us now give a definition for a set $X$ as a general topological space, and then explain how we can generate such a topological space through the so called basis elements or a base for the topology. For more detailed information about general topology one can refer to $[2,7]$.

Definition 1 A topological structure, or just a topology, on a set $X$ is given by a collection $\tau \subset$ $\mathcal{P}(X)$ of subsets of $X$, each called open set and satisfying the following three axioms :

1. $\emptyset \in \tau$ and $X \in \tau$.

2. $\left(\forall \tau_{0}\right)\left(\tau_{0} \subset \tau\right.$ and $\tau_{0}$ is finite $\left.\Rightarrow \cap \tau_{0} \in \tau\right)$.

3. $\left(\forall \tau_{1}\right)\left(\tau_{1} \subset \tau \Rightarrow \cup \tau_{1} \in \tau\right)$.

We call the pair $(X, \tau)$ a topology in $X$, and sometimes the underlying set $X$ can be omitted. The complement of any open subset in regard to $X$ is called closed set.

For any point $x \in X$ and any $A \subset X, A$ is called a neighborhood of $X$ if there is a $A_{0} \in \tau$, such that $x \in A_{0} \subset A$. 
Let us now take a collection $\mathcal{B} \subset \mathcal{P}(X)$ of subsets of $X$, where the following statements hold [7]:

- $\cup \mathcal{B}=X$, which exactly means that

$(\forall x \in X)(\exists B \in \mathcal{B})(x \in B)$

- $\left(\forall B_{1}, B_{2} \in \mathcal{B}\right)(\forall x \in X)$

$\left(x \in B_{1} \cap B_{2} \Rightarrow\left(\exists B_{3} \in \mathcal{B}\right)\left(x \in B_{3} \subset B_{1} \cap B_{2}\right)\right)$.

Definition 2 (cf. [2, Definition 1.5. and 1.6])

The above described collection $\mathcal{B}$ is called base (and its elements are called basis elements) for a special topology, named the topology $\left(X, \tau_{\mathcal{B}}\right)$ generated by $\mathcal{B}$, which is obtained by defining the open sets to be the union of the empty set and the unions of basis elements:

$$
\tau_{\mathcal{B}}=\left\{\cup \tau_{0} \mid \tau_{0} \subset \mathcal{B} .\right\}
$$

Many interesting properties of the basis sets and a detailed proof that the above defined $\tau_{\mathcal{B}} \subset \mathcal{P}(X)$ is actually a topology in $X$ is given in [2, p. 30], for instance. A direct consequence of the set theoretical operations and the above definitions is the following theorem.

Theorem 1 (cf. [2, Theorem 1.9., p. 32]) Let $X$ be a set and $\mathcal{B}$ be a base for the topology $\tau_{\mathcal{B}}$. Then $U$ is open in $\tau_{\mathcal{B}}$ iff for all $x \in U$ there exists a basis element $B_{x} \in \mathcal{B}$ such that $x \in B_{x} \subset U$.

A metric (topological) space can be thought as a very basic space that satisfies a few axioms. The ability to measure and compare distances between elements of a set is often crucial, and it provides more structure than general topological space possesses. When we refer to the elements or "points" of the underlying set, we do not necessarily refer to geometrical points, although this is how most of us usually visualize them. They may be objects of any type, such as sequences, functions, images, sounds, signals, decisions, etc.

Definition 3 A metric on a set $X$ is a function $d: X \times X \rightarrow \mathbb{R}$ with the following properties:

1. $d(x, y) \geq 0$ for all $x, y \in X$, and equality holds iff $x=y$.

2. $d(x, y)=d(y, x)$ for all $x, y \in X$ (symmetry).

3. $d(x, z) \leq d(x, y)+d(y, z)$ for all $x, y, z \in X$ (the triangle inequality).

We call $d(x, y)$ the distance between $x$ and $y$, and the pair $(X, d)$ a metric space.

It is evident that $d$ has the properties we expect when we measure distance between points in rigid geometry. Let us now introduce the three most popular metrics in $R^{n}$, for any positive number $n$.

Definition 4 Taking any $x=\left(x_{1}, \ldots, x_{n}\right), y=$ $\left(y_{1}, \ldots, y_{n}\right) \in R^{n}$, let us define:

\section{Euclidean metric:}

$$
d_{2}(x, y)=\sqrt{\sum_{i=1}^{n}\left(x_{i}-y_{i}\right)^{2}}
$$

2. Manhattan (Hamming) metric:

$$
d_{1}(x, y)=\sum_{i=1}^{n}\left|x_{i}-y_{i}\right|
$$

3. Chebychev (max) metric:

$$
d_{\infty}(x, y)=\max \left\{\left|x_{i}-y_{i}\right|: i \in \overline{1, n}\right\}
$$

Definition 5 Let $(X, d)$ be a metric space. Then the d-open ball (briefly, open ball) of radius $\varepsilon$, centered in $x \in X$, is defined by:

$$
\mathcal{B}(x, d, \varepsilon)=\{y \mid y \in X \& d(x, y)<\varepsilon\}
$$

and the corresponding closed ball is defined by:

$$
\overline{\mathcal{B}}(x, d, \varepsilon)=\{y \mid y \in X \& d(x, y) \leq \varepsilon\} .
$$

Examples of the basis sets (balls) centered at the origin with equal radius for the three already defined metrics on $\mathbb{R}^{2}$ are shown in Fig. 1 .

Definition 6 Let us take $\mathbb{R}^{2}$ as universe and $\xi_{1}, \xi_{2}>0$, then the open $\left(\xi_{1}, \xi_{2}\right)$-neighbourhood, centered in $x=\left(x_{1}, x_{2}\right) \in \mathbb{R}^{2}$, is defined by:

$$
\begin{gathered}
\mathcal{B}(x, \xi) \\
=\left\{y\left|y \in \mathbb{R}^{2} \&\right| x_{1}-y_{1}\left|<\xi_{1} \&\right| x_{2}-y_{2} \mid<\xi_{2}\right\},
\end{gathered}
$$

and the corresponding closed $\left(\xi_{1}, \xi_{2}\right)$-neighbourhood is defined by:

$$
\begin{gathered}
\overline{\mathcal{B}}(x, \xi) \\
=\left\{y\left|y \in \mathbb{R}^{2} \&\right| x_{1}-y_{1}\left|\leq \xi_{1} \&\right| x_{2}-y_{2} \mid \leq \xi_{2}\right\} .
\end{gathered}
$$

Let us formulate the following interesting theorem, relying on Theorem 1 . The reader can easily verify it or refer to $[2,7,9]$.

Theorem 2 Every metric space $(X, d)$ is topological space, when taking the set of its open balls as a basis and therefore the open sets are exactly those, which can be represented as union of d-open balls.

\section{Intuitionistic Fuzzy Sets and Distances}

Let a set $X$ be fixed. An IFS $A$ in $X$ is an object of the following form (see, e.g. $[3,4]$ ):

$$
A=\left\{\left\langle x, \mu_{A}(x), \nu_{A}(x)\right\rangle \mid x \in X\right\},
$$

where the functions $\mu_{A}: X \rightarrow[0,1]$ and $\nu_{A}: X \rightarrow$ $[0,1]$ define the degree of membership and the degree of non-membership of the element $x \in X$, respectively, and for every $x \in X$ :

$$
0 \leq \mu_{A}(x)+\nu_{A}(x) \leq 1 .
$$

Sometimes, for simplicity, we will denote $f_{A}=$ $\left(\mu_{A}, \nu_{A}\right)$ and $f_{A}(x)=\left(\mu_{A}(x), \nu_{A}(x)\right)$, respectively. One very intuitive and practical representation of 


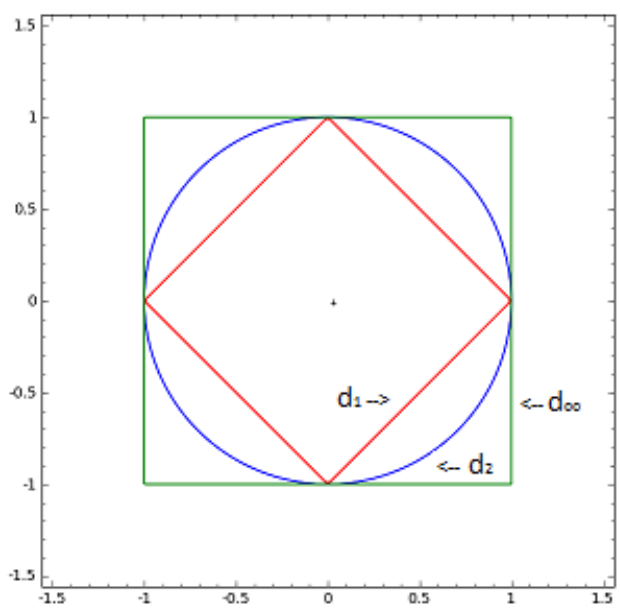

Figure 1: The basis elements, i.e. the open balls of equal radius, in regard of the metrics $\left(\mathbb{R}^{2}, d_{2}\right),\left(\mathbb{R}^{2}, d_{1}\right),\left(\mathbb{R}^{2}, d_{\infty}\right)$.

the IFSs is the triangular representation shown on Fig. 2 .

An additional concept for each IFS in $X$, that is an obvious result of (1) and (2), is called

$$
\pi_{A}(x)=1-\mu_{A}(x)-\nu_{A}(x)
$$

a degree of uncertainty of $x \in A$. It expresses a lack of knowledge of whether $x$ belongs to $A$ or not (cf. [3]). It is obvious that $0 \leq \pi_{A}(x) \leq 1$, for each $x \in X$. Uncertainty degree turns out to be relevant for both the applications and the development of theory of IFSs.

Distances between IFSs are calculated in the literature in two ways, using two parameters only (e.g., $[3-5,13])$ or all three parameters $([6,10-14])$ describing the elements belonging to the sets. Both ways correctly define a metric space, that is, the three axioms for distance are satisfied. Assessing the results obtained by the two ways, one cannot say that both are equal. In $[11,12,14]$, it is shown why in the calculation of distances between IFSs one should prefer all three parameters describing IFSs. Examples of the distances between any two IFSs $A$ and $B$ in $X=\left\{x_{1}, x_{2}, \ldots, x_{n}\right\}$ while using three parameter representation can be found in $[11,12,14]$. A normalized distance or normalized metric $d$ in $X$ is a metric such that $d: X \times X \rightarrow[0,1] \subset \mathbb{R}_{\geq 0}$. Sometimes it is more convenient and easier to work with normalized metrics. Every metric can be normalized (cf. [2]). For a more generalized notion of distances between IFSs (the so called Modified weighted Hausdorff distance - MWHD) the reader may refer to [8].

Let us give the definition of the main metrics in IFSs.

- the Hamming distance (Szmidt and Kacprzyk form):

$$
\begin{aligned}
& l_{3, \mathrm{IFS}}(A, B)=\sum_{i=1}^{n}\left(\left|\mu_{A}\left(x_{i}\right)-\mu_{B}\left(x_{i}\right)\right|\right. \\
& \left.+\left|\nu_{A}\left(x_{i}\right)-\nu_{B}\left(x_{i}\right)\right|+\left|\pi_{A}\left(x_{i}\right)-\pi_{B}\left(x_{i}\right)\right|\right)
\end{aligned}
$$

- the Euclidean distance (Szmidt and Kacprzyk form):

$$
\begin{aligned}
& e_{3, \mathrm{IFS}}(A, B)=\left(\sum_{i=1}^{n}\left(\mu_{A}\left(x_{i}\right)-\mu_{B}\left(x_{i}\right)\right)^{2}\right. \\
& \left.+\left(\nu_{A}\left(x_{i}\right)-\nu_{B}\left(x_{i}\right)\right)^{2}+\left(\pi_{A}\left(x_{i}\right)-\pi_{B}\left(x_{i}\right)\right)^{2}\right)^{\frac{1}{2}}
\end{aligned}
$$

Both distances, corresponding to the two former, are from the interval $[0,1]$. That is they are their corresponding normalized distances.

- the normalized Hamming distance:

$$
L_{3, \mathrm{IFS}}(A, B)=\frac{1}{2 n} l_{3, \mathrm{IFS}}(A, B)
$$

- the normalized Euclidean distance:

$$
E_{3, \mathrm{IFS}}(A, B)=\sqrt{\frac{1}{2 n}} e_{3, \mathrm{IFS}}(A, B)
$$

The counterparts of the above distances while using the first two parameter representation of IFSs are given, e.g., in [3]:

- the Hamming distance:

$$
\begin{aligned}
& l_{2, \operatorname{IFS}}(A, B)=\sum_{i=1}^{n}\left(\left|\mu_{A}\left(x_{i}\right)-\mu_{B}\left(x_{i}\right)\right|\right. \\
& \left.+\left|\nu_{A}\left(x_{i}\right)-\nu_{B}\left(x_{i}\right)\right|\right)
\end{aligned}
$$

- the Euclidean distance :

$$
\begin{aligned}
& e_{2, \mathrm{IFS}}(A, B)=\left(\sum_{i=1}^{n}\left(\mu_{A}\left(x_{i}\right)-\mu_{B}\left(x_{i}\right)\right)^{2}\right. \\
& \left.+\left(\nu_{A}\left(x_{i}\right)-\nu_{B}\left(x_{i}\right)\right)^{2}\right)^{\frac{1}{2}}
\end{aligned}
$$

The normalized distances of the above two are stated as follows.

- the normalized Hamming distance:

$$
L_{2, \mathrm{IFS}}(A, B)=\frac{1}{2 n} l_{2, \mathrm{IFS}}(A, B)
$$

- the normalized Euclidean distance:

$$
E_{2, \mathrm{IFS}}(A, B)=\sqrt{\frac{1}{2 n}} e_{2, \mathrm{IFS}}(A, B)
$$

\section{Intuitionistic Fuzzy Neighbourhoods}

Let us now define a new notion of neighbourhood in terms of the intuitionistic fuzzy sets.

Definition 7 Let us take universe $X, A, B \in$ $\operatorname{IFS}(X)$ and $\varepsilon \in[0,1]$. We shall say that:

- $\mathcal{B}_{I F}\left(A, d_{j}, \varepsilon\right)=\{B \mid B \in \operatorname{IFS}(X) \&$ $\left.(\forall x \in X)\left(d_{j}\left(f_{A}(x), f_{B}(x)\right)<\varepsilon\right)\right\}$ is open IFball with center $A$ and radius $\varepsilon$ (see Definition 5). 
- $\overline{\mathcal{B}_{I F}}\left(A, d_{j}, \varepsilon\right)=\{B \mid B \in \operatorname{IFS}(X) \&$ $\left.(\forall x \in X)\left(d_{j}\left(f_{A}(x), f_{B}(x)\right) \leq \varepsilon\right)\right\}$ is closed IF-ball with center $A$ and radius $\varepsilon$ (see Definition 5).

Analogically let us define the open and closed IFneighbourhoods.

Definition 8 Let us take universe $X, A, B \in$ $\operatorname{IFS}(X)$ and $\xi, \eta \in[0,1]$.

- $\mathcal{B}_{I F}(A, \xi, \eta)=\{B \mid B \in \operatorname{IFS}(X) \&$ $\left.(\forall x \in X)\left(f_{A}(x) \in \mathcal{B}\left(f_{B}(x), \xi, \eta\right)\right)\right\}$ is closed $(\xi, \eta)$-IF-neighbourhood of $A$ (see Definition. 6)

- $\overline{\mathcal{B}_{I F}}(A, \xi, \eta)=\{B \mid B \in \operatorname{IFS}(X) \&$ $\left.(\forall x \in X)\left(f_{A}(x) \in \overline{\mathcal{B}}\left(f_{B}(x), \xi, \eta\right)\right)\right\}$ is open $(\xi, \eta)$-IF-neighbourhood of $A$ (see Definition. 6)

We give now some relations between the standard neighbourhoods and the IF-neighbourhoods. When we write neighbourhood, sometimes we understand by this notion the open (closed) balls too since they are neighbourhood of a special kind.

It turns out that the newly introduced notions of IF-neighbourhoods are more sensitive to large variations in points from the universe (comparing two IFSs) in comparison to the neighbourhoods defined through the distances in IFSs. That is, in the case of the standard neighbourhood or ball, if in at least one point $x_{0} \in X$ for the IFSs $A, B \in \operatorname{IFS}(X)$ we have that $d\left(f_{A}\left(x_{0}\right), f_{B}\left(x_{0}\right)\right)$ (where $d$ is some metric in $\mathbb{R}$ ) is large, then the minimal IF-neighbourhood centered in $A$ and containing $B$ would be large too. This is demonstrated in the next theorems.

In what follows, we take $X$ to be some finite universe and $d_{1}, d_{2}, d_{\infty}$ are the same metrics in $\mathbb{R}^{2}$ as stated in Section 2.

Theorem 3 Let us take $\varepsilon \in[0,1]$. Then the following relations between standard open balls and IFballs for IFSs hold.

1. If $B \in \mathcal{B}_{I F}\left(A, d_{1}, \varepsilon\right)$ then $L_{2, I F S}(A, B)<\frac{\varepsilon}{2}$ i.e. $\mathcal{B}_{I F}\left(A, d_{1}, \varepsilon\right) \subseteq \mathcal{B}\left(A, L_{2, I F S}, \frac{\varepsilon}{2}\right)$

2. If $B \in \mathcal{B}_{I F}\left(A, d_{\infty}, \varepsilon\right)$ then $L_{2, I F S}(A, B)<\varepsilon$ i.e. $\mathcal{B}_{I F}\left(A, d_{\infty}, \varepsilon\right) \subseteq \mathcal{B}\left(A, L_{2, I F S}, \varepsilon\right)$

And analogically, the same statements for standard closed balls and IF-balls for IFSs hold.

3. If $B \in \overline{\mathcal{B}_{I F}}\left(A, d_{1}, \varepsilon\right)$ then $L_{2, I F S}(A, B) \leq \frac{\varepsilon}{2}$ i.e. $\overline{\mathcal{B}_{I F}}\left(A, d_{1}, \varepsilon\right) \subseteq \overline{\mathcal{B}}\left(A, L_{2, I F S}, \frac{\varepsilon}{2}\right)$

4. If $\overline{B \in \mathcal{B}_{I F}}\left(A, d_{\infty}, \varepsilon\right)$ then $L_{2, I F S}(A, B) \leq \varepsilon$ i.e. $\overline{\mathcal{B}_{I F}}\left(A, d_{\infty}, \varepsilon\right) \subseteq \overline{\mathcal{B}}\left(A, L_{2, I F S}, \varepsilon\right)$

Proof: Let us prove the first point only, since the proof of the next three is analogical.

Remind the definition $L_{2, \operatorname{IFS}}(A, B)=$ $\frac{1}{2 n} \sum_{i=1}^{n}\left(\left|\mu_{A}\left(x_{i}\right)-\mu_{B}\left(x_{i}\right)\right|+\left|\nu_{A}\left(x_{i}\right)-\nu_{B}\left(x_{i}\right)\right|\right)$. And since $B \in \mathcal{B}_{\mathrm{IF}}\left(A, d_{1}, \varepsilon\right)$ we have that
$\left|\mu_{A}\left(x_{i}\right)-\mu_{B}\left(x_{i}\right)\right|+\left|\nu_{A}\left(x_{i}\right)-\nu_{B}\left(x_{i}\right)\right|<\varepsilon$ for any $x_{i} \in X$. Therefore, $L_{2, \mathrm{IFS}}(A, B) \leq \frac{1}{2 n}(n \varepsilon)=\frac{1}{2} \varepsilon$.

Let us now state the relations between standard neighbourhoods and IF-neighbourhoods. Since the proof of the next theorem is similar to Theorem 3, it is left for the reader.

Theorem 4 Let us take $\xi, \eta \in[0,1]$. Then the following relations between standard open (closed) neighbourhoods and open (closed) IF-balls with respect to $L_{2, I F S}$ hold.

1. If $B \in \mathcal{B}_{I F}(A, \xi, \eta)$ then $L_{2, I F S}(A, B)<\frac{\xi+\eta}{2}$ i.e. $\mathcal{B}_{I F}(A, \xi, \eta) \subseteq \mathcal{B}\left(A, L_{2, I F S}, \frac{\xi+\eta}{2}\right)$

2. If $B \in \overline{\mathcal{B}_{I F}}(A, \xi, \eta)$ then $L_{2, I F S}(A, B) \leq \frac{\xi+\eta}{2}$ i.e. $\overline{\mathcal{B}_{I F}}(A, \xi, \eta) \subseteq \overline{\mathcal{B}}\left(A, L_{2, I F S}, \frac{\xi+\eta}{2}\right)$

Theorem 5 For any $A, B \in \operatorname{IFS}(X)$ the following inequality hold.

$$
L_{3, I F S}(A, B) \leq 2 L_{2, I F S}(A, B)
$$

Proof: Obviously, for any two numbers $a, b \in \mathbb{R}$ we have that $|a+b| \leq|a|+|b|$. Therefore, $\left|\pi_{A}\left(x_{i}\right)-\pi_{B}\left(x_{i}\right)\right|=\mid \mu_{B}\left(x_{i}\right)$

$-\mu_{A}\left(x_{i}\right)+\nu_{B}\left(x_{i}\right)-\nu_{A}\left(x_{i}\right)|\leq| \mu_{B}\left(x_{i}\right)-$ $\mu_{A}\left(x_{i}\right)|+| \nu_{B}\left(x_{i}\right)-\nu_{A}\left(x_{i}\right) \mid$. The last two statements provide that $L_{3, \mathrm{IFS}}(A, B) \leq$ $\frac{1}{2 n} \sum_{i=1}^{n} 2\left(\left|\mu_{A}\left(x_{i}\right)-\mu_{B}\left(x_{i}\right)\right|+\left|\nu_{A}\left(x_{i}\right)-\nu_{B}\left(x_{i}\right)\right|\right)$, which implies that $L_{3, \mathrm{IFS}}(A, B) \leq 2 L_{2, \mathrm{IFS}}(A, B)$. The theorem is proved.

The last theorem provides that for any $\varepsilon>0$ we have that

$$
\mathcal{B}\left(A, L_{2, \mathrm{IFS}}, \varepsilon\right) \subseteq \mathcal{B}\left(A, L_{3, \mathrm{IFS}}, 2 \varepsilon\right)
$$

Theorem 6 For any $\varepsilon, \xi, \eta \in[0,1]$ the following theoretical set inclusions hold.

1. $\mathcal{B}_{I F}\left(A, d_{1}, \varepsilon\right) \subseteq \mathcal{B}\left(A, L_{3, I F S}, \varepsilon\right)$ (see Theorem 3)

2. $\mathcal{B}_{I F}\left(A, d_{\infty}, \varepsilon\right) \subseteq \mathcal{B}\left(A, L_{3, I F S}, 2 \varepsilon\right)$ (see Theorem 3)

3. $\mathcal{B}_{I F}(A, \xi, \eta) \subseteq \mathcal{B}\left(A, L_{3, I F S}, \xi+\eta\right)$ (see Theorem 4)

Through the following theorem, we give some relations between IF-balls in respect to $E_{2, \text { IFS }}$ and the standard balls and standard $\xi, \eta$-neighbourhoods.

Theorem 7 Let us take $\varepsilon, \xi, \eta \in[0,1]$. Then the following statements hold.

1. If $B \in \mathcal{B}_{I F}\left(A, d_{2}, \varepsilon\right)$ then $E_{2, I F S}(A, B)<\frac{\varepsilon}{\sqrt{2}}$ or $\mathcal{B}_{I F}\left(A, d_{2}, \varepsilon\right) \subseteq \mathcal{B}\left(A, E_{2, I F S}, \frac{\varepsilon}{\sqrt{2}}\right)$

2. If $B \in \mathcal{B}_{I F}\left(A, d_{\infty}, \varepsilon\right)$ then $E_{2, I F S}(A, B)<\varepsilon$ i.e. $\mathcal{B}_{I F}\left(A, d_{\infty}, \varepsilon\right) \subseteq \mathcal{B}\left(A, E_{2, I F S}, \varepsilon\right)$

3. If $B \in \mathcal{B}_{I F}(A, \xi, \eta)$ then $E_{2, I F S}(A, B)<$ $\sqrt{\frac{\xi^{2}+\eta^{2}}{2}}$

i.e. $\mathcal{B}_{I F}(A, \xi, \eta) \subseteq \mathcal{B}\left(A, E_{2, I F S}, \sqrt{\frac{\xi^{2}+\eta^{2}}{2}}\right)$

Let us now state some relations between $E_{3, \text { IFS }}$ and the IF-balls and IF-neighbourhoods. 
Theorem 8 For any $\varepsilon, \xi, \eta \in[0,1]$ the following statements hold.

1. If $B \in \mathcal{B}_{I F}\left(A, d_{\infty}, \varepsilon\right)$ then $E_{3, I F S}(A, B)<\sqrt{3} \varepsilon$ i.e. $\mathcal{B}_{I F}\left(A, d_{\infty}, \varepsilon\right) \subseteq \mathcal{B}\left(A, E_{3, I F S}, \sqrt{3} \varepsilon\right)$

2. If $B \in \mathcal{B}_{I F}(A, \xi, \eta)$ then $E_{3, I F S}(A, B)<$ $\sqrt{\xi^{2}+\eta^{2}+\xi \eta}$ i.e.

$\mathcal{B}_{I F}\left(A, d_{\infty}, \varepsilon\right) \subseteq \mathcal{B}\left(A, E_{3, I F S}, \sqrt{\xi^{2}+\eta^{2}+\xi \eta}\right)$

Proof: We prove 2. since 1 . is analogical.

We have that $\pi_{A}\left(x_{i}\right)-\pi_{B}\left(x_{i}\right)=\mu_{B}\left(x_{i}\right)-\mu_{A}\left(x_{i}\right)+$ $\nu_{B}\left(x_{i}\right)-\nu_{A}\left(x_{i}\right)$ and therefore $\left(\pi_{A}\left(x_{i}\right)-\pi_{B}\left(x_{i}\right)\right)^{2}=$ $\left(\mu_{B}\left(x_{i}\right)-\mu_{A}\left(x_{i}\right)\right)^{2}+\left(\nu_{B}\left(x_{i}\right)-\nu_{A}\left(x_{i}\right)\right)^{2}+2\left(\mu_{B}\left(x_{i}\right)-\right.$ $\left.\mu_{A}\left(x_{i}\right)\right)\left(\nu_{B}\left(x_{i}\right)-\nu_{A}\left(x_{i}\right)\right) \leq \xi^{2}+\eta^{2}+2 \xi \eta$. From (3) and (4), we have $E_{3, \mathrm{IFS}}(A, B)=\left(\frac{1}{2 n} \sum_{i=1}^{n}\left(\mu_{A}\left(x_{i}\right)-\right.\right.$ $\left.\left.\mu_{B}\left(x_{i}\right)\right)^{2}+\left(\nu_{A}\left(x_{i}\right)-\nu_{B}\left(x_{i}\right)\right)^{2}+\left(\pi_{A}\left(x_{i}\right)-\pi_{B}\left(x_{i}\right)\right)^{2}\right)^{\frac{1}{2}}$ hence,

$$
E_{3, \mathrm{IFS}}(A, B) \leq \sqrt{\frac{1}{2 n}} \sqrt{2 n\left(\xi^{2}+\eta^{2}+\xi \eta\right)},
$$

which completes the proof.

\section{Applications of the IF-neighbourhoods to Modal and Extended Modal Operators over IFSs}

For every IFS $A$ a lot of operators are defined (see, e.g. $[3,4])$, the most important of which, related to the applications of the present research, are $(\alpha, \beta \in$ $[0,1])$ the standard modal IF operators

$$
\begin{aligned}
& \square A \quad=\left\{\left\langle x, \mu_{A}(x), 1-\mu_{A}(x)\right\rangle \mid x \in X\right\} ; \\
& \diamond A \quad=\quad\left\{\left\langle x, 1-\nu_{A}(x), \nu_{A}(x)\right\rangle \mid x \in X\right\} ; \\
& F_{\alpha, \beta}(A)=\left\{\left\langlex, \mu_{A}(x)+\alpha \pi_{A}(x), \nu_{A}(x)\right.\right. \\
& \left.\left.+\beta \pi_{A}(x)\right\rangle \mid x \in X\right\} \text {, } \\
& \text { where } \alpha+\beta \leq 1 \text {; } \\
& G_{\alpha, \beta}(A)=\left\{\left\langle x, \alpha \mu_{A}(x), \beta \nu_{A}(x)\right\rangle \mid x \in X\right\} ; \\
& \begin{aligned}
H_{\alpha, \beta}(A)= & \left\{\left\langle x, \alpha \mu_{A}(x), \nu_{A}(x)+\beta \pi_{A}(x)\right\rangle\right. \\
& \mid x \in X\} ;
\end{aligned} \\
& H_{\alpha, \beta}^{*}(A)=\left\{\left\langlex, \alpha \mu_{A}(x), \nu_{A}(x)+\beta(1-\right.\right. \\
& \left.\left.\left.\alpha \mu_{A}(x)-\nu_{A}(x)\right)\right\rangle \mid x \in X\right\} \text {; } \\
& \begin{aligned}
J_{\alpha, \beta}(A)= & \left\{\left\langle x, \mu_{A}(x)+\alpha \pi_{A}(x), \beta \nu_{A}(x)\right\rangle\right. \\
& \mid x \in X\} ;
\end{aligned} \\
& J_{\alpha, \beta}^{*}(A)=\left\{\left\langlex, \mu_{A}(x)+\alpha\left(1-\mu_{A}(x)\right.\right.\right. \\
& \left.\left.\left.-\beta \nu_{A}(x)\right), \beta \nu_{A}(x)\right\rangle \mid x \in X\right\} \text {; } \\
& C(A) \quad=\quad\{\langle x, K, L\rangle \mid x \in X\} \\
& I(A) \quad=\{\langle x, k, l\rangle \mid x \in X\} .
\end{aligned}
$$

where

$$
K_{A}=\sup _{y \in X} \mu_{A}(y), L_{A}=\inf _{y \in X} \nu_{A}(y)
$$

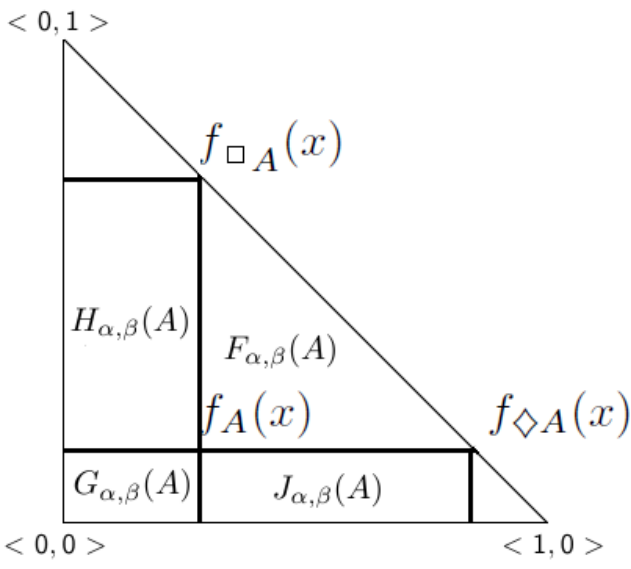

Figure 2: Triangular representation of $A \in \operatorname{IFS}(X)$ in a particular point $x \in X$, where $f_{A}(x)$ stands for the point with coordinates $\left(\mu_{A}(x), \nu_{A}(x)\right)$.

$$
k_{A}=\inf _{y \in X} \mu_{A}(y), l_{A}=\sup _{y \in X} \nu_{A}(y)
$$

On Fig. 2 one may see the regions of operation of the extended modal operators.

Let us take,

$$
P_{A}=\sup _{y \in X} \pi_{A}(y), p_{A}=\inf _{y \in X} \pi_{A}(y)
$$

We shall call the IFSs $A$ and $B$ " $(\xi, \eta)$ neighbouring" when:

$$
\left\{\begin{array}{l}
\sup _{y \in X}\left|\mu_{A}(x)-\mu_{B}(x)\right| \leq \xi \\
\inf _{y \in X}\left|\nu_{A}(x)-\nu_{B}(x)\right| \leq \eta
\end{array}\right.
$$

In other words, $A$ and $B$ are " $(\xi, \eta)$-neighbouring" if and only if $B \in \mathcal{B}_{\mathrm{IF}}(A, \xi, \eta)$.

Theorem 9 For every IFS $A$ and for every $\xi, \eta \in$ $[0,1]$ :

1. $A$ and $F_{\alpha, \beta}(A)$ are $(\xi, \eta)$-neighbouring iff $0 \leq$ $\alpha \leq \min \left(1, \frac{\xi}{P_{A}}\right)$ and $0 \leq \beta \leq \min \left(1, \frac{\eta}{P_{A}}\right)$, where $\alpha+\beta<1$;

2. $A$ and $G_{\alpha, \beta}(A)$ are $(\xi, \eta)$-neighbouring iff $\max \left(0,1-\frac{\xi}{K_{A}}\right) \leq \alpha \leq 1$ and $\max \left(0,1-\frac{\eta}{l_{A}}\right) \leq$ $\beta \leq 1$

3. $A$ and $H_{\alpha, \beta}(A)$ are $(\xi, \eta)$-neighbouring iff $\max \left(0,1-\frac{\xi}{K_{A}}\right) \leq \alpha \leq 1$ and $0 \leq \beta \leq$ $\min \left(1, \frac{\eta}{P_{A}}\right)$;

4. $A$ and $J_{\alpha, \beta}(A)$ are $(\xi, \eta)$-neighbouring iff $0 \leq$ $\alpha \leq \min \left(1, \frac{\xi}{P_{A}}\right)$ and $\max \left(0,1-\frac{\eta}{l_{A}}\right) \leq \beta \leq 1$;

5. $A$ and $H_{\alpha, \beta}^{*}(A)$ are $(\xi, \eta)$-neighbouring iff $\max \left(0,1-\frac{\xi}{K_{A}}\right) \leq \alpha \leq 1$ and $0 \leq \beta \leq$ $\min \left(1, \frac{\eta}{1-\max \left(0,1-\frac{\xi}{k_{A}}\right) \mu_{A}\left(x^{*}\right)-\nu_{A}\left(x^{*}\right)}\right)$;

6. $A$ and $J_{\alpha, \beta}^{*}(A)$ are $(\xi, \eta)$-neighbouring iff $0 \leq$ $\alpha \leq \min \left(1, \frac{\xi}{1-\mu_{A}\left(x^{*}\right)-\max \left(0,1-\frac{\eta}{L_{A}}\right) \nu_{A}\left(x^{*}\right)}\right)$ and $\max \left(0,1-\frac{\eta}{l_{A}}\right) \leq \beta \leq 1$, where $x^{*} \in X$ satisfies the equality $\mu_{A}\left(x^{*}\right)+\nu_{A}\left(x^{*}\right)=1-p_{A}$. 
Proof: 1. Let $A$ and $F_{\alpha, \beta}(A)$ be $(\xi, \eta)$-neighbouring. Therefore, for every $x \in X: \mid \mu_{A}(x)+\alpha \pi_{A}(x)-$ $\mu_{A}(x) \mid \leq \xi$ and $\left|\nu_{A}(x)+\beta \pi_{A}(x)-\nu_{A}(x)\right| \leq \eta$, i.e. $\alpha \pi_{A}(x) \leq \xi$ and $\beta \pi_{A}(x) \leq \eta$. Hence $\alpha P_{A} \leq \xi$ and $\beta P_{A} \leq \eta$, but $\alpha+\beta \in[0,1]$. Therefore $\alpha \leq$ $\min \left(1, \frac{\xi}{P_{A}}\right)$ and $\beta \leq \min \left(1, \frac{\eta}{P_{A}}\right)$.

In the opposite case, let $\alpha$ and $\beta$ satisfy the above conditions and let $\alpha+\beta \in[0,1]$. Therefore for every $x \in X: 0 \leq \alpha \leq \frac{\xi}{P_{A}} \leq \frac{\xi}{\pi_{A}(x)}$ and $0 \leq \beta \leq \frac{\eta}{P_{A}} \leq$ $\frac{\eta}{\pi_{A}(x)}$.

Therefore $\left|\mu_{A}(x)+\alpha \pi_{A}(x)-\mu_{A}(x)\right|=\alpha \pi_{A}(x) \leq$ $\pi_{A}(x) \min \left(1, \frac{\xi}{P_{A}}\right)=\min \left(\pi_{A}(x), \frac{\xi \pi_{A}(x)}{P_{A}}\right) \leq$ $\min \left(\pi_{A}(x), \xi\right) \leq \xi$ and $\mid \nu_{A}(x)+\beta \pi_{A}(x)-$ $\nu_{A}(x) \mid=\beta \pi_{A}(x) \leq \pi_{A}(x) \min \left(1, \frac{\eta}{P_{A}}\right)=$ $\min \left(\pi_{A}(x), \frac{\eta \pi_{A}(x)}{P_{A}}\right) \leq \min \left(\pi_{A}(x), \eta\right) \leq \eta$.

\section{2. -6 . can be proved analogically.}

Now we shall discuss the opposite problem and we skip the proof since it is trivial as in the previous theorem.

Theorem 10 For every IFS $A$ and for every $\alpha, \beta \in$ $[0,1]$ :

1. $A$ and $F_{\alpha, \beta}(A)$ are $(\xi, \eta)$-neighbouring iff $\alpha P_{A} \leq \xi$ and $\beta P_{A} \leq \eta$, where $\alpha+\beta \leq 1$;

2. $A$ and $G_{\alpha, \beta}(A)$ are $(\xi, \eta)$-neighbouring iff (1$\alpha) K_{A} \leq \xi$ and $(1-\beta) l_{A} \leq \eta$

3. $A$ and $H_{\alpha, \beta}(A)$ are $(\xi, \eta)$-neighbouring iff (1$\alpha) \cdot K_{A} \leq \xi$ and $\beta P_{A} \leq \eta$;

4. $A$ and $J_{\alpha, \beta}(A)$ are $(\xi, \eta)$-neighbouring iff $\alpha P_{A} \leq \xi$ and $(1-\beta) l_{A} \leq \eta$

5. $A$ and $H_{\alpha, \beta}^{*}(A)$ are $(\xi, \eta)$-neighbouring iff $(1-$ $\alpha) K_{A} \leq \varepsilon$ and $\beta\left(1-\alpha \mu_{A}\left(x^{*}\right)-\nu_{A}\left(x^{*}\right)\right) \leq \eta$;

6. $A$ and $J_{\alpha, \beta}^{*}(A)$ are $(\xi, \eta)$-neighbouring iff $\alpha(1-$ $\left.\mu_{A}\left(x^{*}\right)-\beta \cdot \nu_{A}\left(x^{*}\right)\right) \leq \xi$ and $(1-\beta) l_{A} \leq \eta$, where $x^{*} \in X$ satisfies the equality $\mu_{A}\left(x^{*}\right)+$ $\nu_{A}\left(x^{*}\right)=1-p_{A}$.

\section{Conclusion}

In summary, we have introduced the concepts of IF-neighbourhood and IF-ball. Many relations between them and the standard notions of neighbourhood and ball in metric spaces have also been stated.

It turns out that the newly introduced IF-neighbourhood is more sensitive to large variations in points from the universe (comparing two IFSs) in comparison to the neighbourhoods defined by the distances in IFSs. That is, in the case of the IFneighbourhood or IF-ball, if in only a few points (at least one) $x_{0} \in X$ for the intuitionistic fuzzy sets $A, B \in \operatorname{IFS}(X)$ we have that $d\left(f_{A}\left(x_{0}\right), f_{B}\left(x_{0}\right)\right)$ (where $d$ is some metric in $\mathbb{R}$ ) is large, then the minimal IF-neighbourhood centered in $A$ and containing $B$ would be large too. Whereas, suppose that only $d\left(f_{A}\left(x_{0}\right), f_{B}\left(x_{0}\right)\right)$ is large for some point $x_{0}$ from the universe, but for the rest of the points from $X, d\left(f_{A}(x), f_{B}(x)\right)$ is very small, in some sense, it may compensate the value of the large scale of
$d\left(f_{A}\left(x_{0}\right), f_{B}\left(x_{0}\right)\right.$ and $L(A, B)$ could be not so large ( $L$ is some metric for IFSs).

We have also proposed applications to the theory, stated in this paper, through the extended modal operators for IFSs.

In a next research of the authors, the introduced concept of IF-neighbourhoods will be used for extending of some procedures related to multicriterial and intercriterial analysis.

\section{Acknowledgments}

The authors are thankful for the support provided by the Bulgarian National Science Fund under Grant Ref. No. DFNI-I-02-5 "InterCriteria Analysis: A New Approach to Decision Making".

\section{References}

[1] A. Ban. Intuitionistic Fuzzy Measures. Theory and Applications. Nova Sci. Publishers, New York, 2006.

[2] C. Adams and R. Franzosa, Introduction to topology - pure and applied. Pearson Prentice Hall, USA, 2008.

[3] K. Atanassov, Intuitionistic Fuzzy Sets: Theory and Applications. Springer-Verlag, Heidelberg, 1999.

[4] K. Atanassov, On Intuitionistic Fuzzy Sets Theory. Springer-Verlag, Berlin, 2012.

[5] K. Atanassov, P. Vassilev, R. Tcvetkov. Intuitionistic Fuzzy Sets, Measures and Integrals. "Prof. M. Drinov" Academic Publishing House, Sofia, 2013.

[6] Deng-Feng Li, Multiattribute decision making models and methods using intuitionistic fuzzy sets. Journal of Computer and System Sciences, 70:73-85, 2005.

[7] K. Kuratowski, Topology Vol 1., Academic Press, New York and London, 1966.

[8] E. Marinov, E. Szmidt, J. Kacprzyk and R. Tcvetkov, A modified weighted Hausdorff distance between intuitionistic fuzzy sets, IEEE Int. Conf. on Intelligent Systems IS'12, 2012

[9] J. Munkres, Topology, 2nd ed., Prentice Hall, 2000.

[10] Y. Narukawa and V. Torra Non-monotonic fuzzy measure and intuitionistic fuzzy set. LNAI 3885:150-160, 2006.

[11] E. Szmidt and J. Baldwin, New similarity measure for intuitionistic fuzzy set theory and mass assignment theory. Notes on Intuitionistic Fuzzy Sets, 9(3):60-76, 2003.

[12] E. Szmidt and J. Baldwin, Entropy for intuitionistic fuzzy set theory and mass assignment theory. Notes on Intuitionistic Fuzzy Sets, 10(3):15-28, 2004.

[13] E. Szmidt, Distances and Similarities in Intuitionistic Fuzzy Sets, Springer-Verlag, Studies in Fuzziness and Soft Computing, 307:2014. 
[14] E. Szmidt and J. Kacprzyk Distances between intuitionistic fuzzy sets. Fuzzy Sets and Systems, 114(3):505-518, 2000.

[15] L. Zadeh, Fuzzy sets. Information and Control, 8:338-353, 1965. 